\title{
Ricordo di Gianni Mombello
}

\section{(2) OpenEdition \\ 1 Journals}

\section{Edizione digitale}

URL: http://journals.openedition.org/studifrancesi/29803

DOI: 10.4000/studifrancesi.29803

ISSN: 2421-5856

\section{Editore}

Rosenberg \& Sellier

\section{Edizione cartacea}

Data di pubblicazione: 1 avril 2006

Paginazione: 3-4

ISSN: 0039-2944

\section{Notizia bibliografica digitale}

«Ricordo di Gianni Mombello», Studi Francesi [Online], 148 (XLX | I) | 2006, online dal 30 novembre 2015, consultato il 18 avril 2021. URL: http://journals.openedition.org/studifrancesi/29803 ; DOI: https://doi.org/10.4000/studifrancesi.29803

\section{(c) $\underset{\mathrm{EY}}{(\mathrm{NQ} \mathrm{NO}}$}

Studi Francesi è distribuita con Licenza Creative Commons Attribuzione - Non commerciale - Non opere derivate 4.0 Internazionale. 


\section{Ricordo di Gianni Mombello}

Il 5 settembre 2005, dopo breve e crudele malattia, Gianni Mombello ci ba lasciati. Il vuoto che si è creato nei nostri animi è incolmabile, la nostalgia del suo profilo umano e del calore della sua amicizia è, per tutti noi, penosa ed acerba. Condirettore di Studi francesi, Gianni amava con dedizione la nostra Rivista, ne difendeva con slancio la fedeltà alle origini, credeva nelle sue ragioni di vita. Il suo lavoro redazionale si svolgeva all'insegna della discrezione e dell'assiduità, dell'umiltà e dello scrupolo. Era tanto più concretamente impegnato quanto meno sbandierava quel che faceva o ricorreva a un inutile e vacuo verbiage. Rideva di quanti si compiacciono di quella che con spirito bonario chiamava la libido ciacolandi. Era convinto che i nostri Studi svolgessero una funzione culturale importante, quella voluta dal loro fondatore, di natura storico-critica, e che costituissero una palestra insostituibile per $i$ giovani ricercatori e studiosi.

$\dot{E}$ inutile dire che la Rivista soffrirà della Sua assenza. E tuttavia, proprio la Sua scomparsa ci spinge a un più costante e instancabile impegno: sarà un modo di rispettare il Suo desiderio di una pertinace, perseverante continuità. Le collaboratrici ed ex-allieve a lui più vicine pensano di commemorarLo adeguatamente raccogliendo in volume $i$ Suoi ultimi scritti. Per ora, ci limitiamo a riprodurre, in apertura di questo fascicolo, quanto già abbiamo detto rivolgendoci a Lui nella premessa che apre gli Studi raccolti e pubblicati in Suo onore in occasione del Suo settantesimo compleanno (Favola, mito ed altri saggi di letteratura e filologia, in onore di Gianni Mombello, Alessandria, Edizioni dell'Orso, 2004).

Ripercorrere le varie epoche della tua vita significa per noi, caro Gianni, non solo ritrovare tutti i momenti della tua intensa attività e le varie fasi del tuo svolgimento intellettuale, ma anche risalire alle varie tappe del nostro caloroso rapporto umano, verificare il continuo rinsaldarsi dell'amicizia che ci ha legati a te. Ritroviamo nel tuo curriculum i tuoi anni di Montmélian, poi quelli di Digione, poi il lungo soggiorno parigino, al CNRS e alla scuola di Lecoy, e nel frattempo la tua fedele vicinanza a Franco Simone, tuo e nostro Maestro, e il tuo continuo operare nel solco del suo insegnamento, e poi Bergamo e poi, finalmente, Torino, e la tua presenza alla direzione di quegli "Studi Francesi" a cui hai sempre dato tanta parte delle tue energie, e poi la tua nomina, proposta dal grande amico Terreaux, all'Académie de Savoie.... Quanti ricordi, quante occasioni, quanti momenti, allora brevi e rapidi, ora eterni, legati ad esempio a quel convegno di Lione, o a quell'altro di Certaldo, o alle comuni presenze e agli incontri alla Réserve della B.N. parigina!

Ma poi ripercorriamo l'ampio ventaglio dei tuoi scritti e vi cogliamo le tracce e i segni delle virtù del tuo animo. Nei tuoi primi saggi su Verlaine (ma anche su Valery Larbaud, su Villiers, su Zola) trovavano spazio, in fondo, la tua vocazione poetica, la voce della tua sensibilità, che tu poi hai voluto in qualche modo soffocare, forse perché ti sembrava troppo manifesta e volevi tenerla segreta, come tua intima e personale ricchezza. Così, sei passato alla filologia, alla linguistica e alla storia della lingua, ad una solida erudizione, ma anche qui le tue doti più vere hanno avuto modo di manifestarsi, il tuo amore, cioè, non tanto per personaggi già noti o di primo piano, quanto per scrittori apparentemente marginali, in realtà ricchissimi di umanità e di cultura: la tua Christine de Pizan innanzi tutto, alla quale hai dedicato lunghe e pazienti ricerche (La tradizione manoscritta dell' "Epistre Othea" di Christine de Pizan, 1967), ma anche Jean Miélot, Claude de Seyssel, Julien Macho, Guillaume Tardif, Pierre Bertelot, Jean Tisserand, Jean Meslier, Pierre Millot, Pierre de La Serre, Antoine Du Moulin, e quell'Alexis Jure a cui dedicavi un'esemplare monografia (Sur les traces d'Alexis Jure de 
Chieri, 1984), e anche gli italiani Nevizzano, Alione, Astemio, insomma gli esponenti singolari di una cultura poco nota e in realtà di così rilevante interesse, una galleria davvero insolita nel panorama scientifico di uno studioso che si è evidentemente proposto come suo scopo primario quello di investigare il sottobosco letterario più fitto e meno esplorato, di riportare alla luce figure dimenticate di una cultura al cui sviluppo hanno contribuito non solo le voci più note, ma anche e soprattutto una coralità di linguaggi più in sordina, di proposte e messaggi, riflessioni e sollecitazioni. Anche delle voci più alte, del resto, hai saputo occuparti, quando ad esempio hai studiato la fortuna delle "tre corone" in quel Quattrocento francese divenuto ormai un tuo specifico campo di ricerca. Poi, a un certo punto della tua carriera di studioso, ti sei chinato su un genere che ti è congeniale e che è così emblematico dei sotterranei percorsi di una creazione letteraria in cui etica e poesia così profondamente si saldano, il genere cioè della favola: hai studiato, così, favole e favolisti, miti e leggende, terreno vasto e complesso in cui hai dato contributi essenziali e hai sollecitato le nuove ricerche condotte dai giovani che rappresentano la tua scuola (Le raccolte francesi di favole esopiane, 1981). Parallelamente hai praticamente "inventato" nuovi filoni di indagine, ad esempio quello relativo al genere dei Noëls, in cui hai dato la misura della tua capacità di scoprire e isolare determinati modi di scrittura poetica, o quello relativo all'epistolografia, scoprendo e dirigendo la pubblicazione del monumentale carteggio inedito di Albert Bailly. Questo senza dire che hai coltivato, naturalmente, anche aspetti della tua specifica disciplina, come quando hai studiato la parola talent pubblicando sul tema una vasta monografia (Les avatars de "Talentum", 1976) o come quando ti sei chinato su carte inedite di quel grande linguista che fu Vaugelas.

Si tratta di contributi di valore, in cui però a noi piace ritrovare, oltre al pregio specifico, le mille risonanze della tua umanità, la serietà del tuo assiduo impegno, la tua pazienza nella ricerca continua di vie infrequentate, il tuo senso della discrezione, della prudenza e, direi, di una cristiana umiltà, il tuo rifiuto, sul piano espressivo, di ogni facile retorica e, sul piano del vivere, di ogni inaccettabile compromesso, il tuo gusto del lavoro segreto e dell'appartato ritiro negli spazi dell'anima. Sono queste le ragioni che ci spingono a dirti, caro Gianni, in modo certo inadeguato, che sempre ti abbiamo ammirato per il tuo rigore scientifico e intellettuale e che ti vogliamo bene per l'esempio costante che ci hai sempre dato di autenticità, di integrità, di nobiltà. 